\title{
Restriction to finite-index subgroups as étale extensions in topology, KK-theory and geometry
}

\author{
PAUL BALMER \\ IVo DELL'AMBrogio \\ BEREN SANDERS
}

For equivariant stable homotopy theory, equivariant KK-theory and equivariant derived categories, we show how restriction to a subgroup of finite index yields a finite commutative separable extension, analogous to finite étale extensions in algebraic geometry.

13B40, 18E30; 55P91, 19K35, 14F05

\section{Introduction and main results}

In linear representation theory of discrete groups, the first-named author proved that restriction to a finite-index subgroup can be realized as a finite étale extension; see Balmer [3, Part I]. (The exact statement is a special case of Theorem 1.3 below.) A priori, this result of [3] seems very module-theoretic in nature. The goal of the present article is horizontal generalization to a broad array of equivariant settings, from topology to analysis. Specifically, we prove the following three results, in which the reader should feel free to assume that the group $G$ is finite, if so inclined.

1.1 Theorem Let $G$ be a compact Lie group and let $H \leq G$ be a closed subgroup of finite index. Then the suspension $G$-spectrum $A_{H}^{G}:=\Sigma^{\infty} G / H_{+}$is a commutative separable ring object in the equivariant stable homotopy category $\mathrm{SH}(G)$. Moreover, there is an equivalence of categories $\mathrm{SH}(H) \cong A_{H}^{G}-\operatorname{Mod}_{\mathrm{SH}(G)}$ between $\mathrm{SH}(H)$ and the category of left $A_{H}^{G}$-modules in $\mathrm{SH}(G)$ under which the restriction functor $\mathrm{SH}(G) \rightarrow \mathrm{SH}(H)$ becomes isomorphic to the extension-of-scalars functor $\mathrm{SH}(G) \rightarrow A_{H}^{G}-\operatorname{Mod}_{\mathrm{SH}(G)}$.

1.2 Theorem Let $G$ be a second-countable locally compact Hausdorff group and let $H \leq G$ be a closed subgroup of finite index. Then the finite-dimensional algebra $A_{H}^{G}:=\mathbb{C}(G / H)$ is a commutative separable ring object in the equivariant Kasparov category $\operatorname{KK}(G)$ of $G-C^{*}$-algebras. Moreover, there is an equivalence of categories 
$\mathrm{KK}(H) \cong A_{H}^{G}-\operatorname{Mod}_{\mathrm{KK}(G)}$ between $\mathrm{KK}(H)$ and the category of left $A_{H}^{G}$-modules in $\mathrm{KK}(G)$ under which the restriction functor $\mathrm{KK}(G) \rightarrow \mathrm{KK}(H)$ becomes isomorphic to the extension-of-scalars functor $\mathrm{KK}(G) \rightarrow A_{H}^{G}-\operatorname{Mod}_{\mathrm{KK}(G)}$.

1.3 Theorem Let $G$ be a discrete group acting on a ringed space $S$ (for instance, a scheme) and let $H \leq G$ be a subgroup of finite index. Then the free $\mathcal{O}_{S}$-module $A_{H}^{G}:=\mathcal{O}_{S}(G / H)$ on $G / H$ is a commutative separable ring object in the derived category $\mathrm{D}(G ; S)$ of $G$-equivariant sheaves of $\mathcal{O}_{S}$-modules. Moreover, there is an equivalence of categories $\mathrm{D}(H ; S) \cong A_{H}^{G}-\operatorname{Mod}_{\mathrm{D}(G ; S)}$ between $\mathrm{D}(H ; S)$ and the category of left $A_{H}^{G}$-modules in $\mathrm{D}(G ; S)$ under which the restriction functor $\mathrm{D}(G ; S) \rightarrow \mathrm{D}(H ; S)$ becomes isomorphic to the extension-of-scalars functor $\mathrm{D}(G ; S) \rightarrow A_{H}^{G}-\operatorname{Mod}_{\mathrm{D}(G ; S)}$.

These theorems are proved in Sections 3, 4 and 5 respectively. In all three cases, the multiplication $\mu: A_{H}^{G} \otimes A_{H}^{G} \rightarrow A_{H}^{G}$ on the ring object $A_{H}^{G}$ is characterized by the rule

$$
\mu\left(\gamma \otimes \gamma^{\prime}\right)=\left\{\begin{array}{ll}
\gamma & \text { if } \gamma=\gamma^{\prime} \\
0 & \text { if } \gamma \neq \gamma^{\prime}
\end{array} \quad \text { for all } \gamma, \gamma^{\prime} \in G / H .\right.
$$

Let us provide some explanations and motivation.

If not familiar with [3], the reader might be surprised to see that restriction can be interpreted as an extension. When we consider a category $\mathscr{C}=\mathscr{C}(G)$ depending on a group $G$, like the above $\operatorname{SH}(G), \operatorname{KK}(G)$ or $\mathrm{D}(G ; S)$, and when $H \leq G$ is a subgroup, the rough intuition is that the H-equivariant category $\mathscr{C}(H)$ should only be a "piece" of the corresponding $G$-equivariant category $\mathscr{C}(G)$. At first, one might naively hope that $\mathscr{C}(H)$ is a localization of $\mathscr{C}(G)$, as a category. Although this naive guess essentially always fails, we are going to prove that this intuition is actually valid if one uses a broader, more flexible notion of "localization". This broader notion is conceptually analogous to localization with respect to the étale topology in algebraic geometry rather than the Zariski topology. Seen from the perspective of Galois theory, it is not so surprising that extension should be connected to restriction to a smaller group.

Let us be more precise. Consider a category $\mathscr{C}$, like our $\mathscr{C}(G)$, equipped with a tensor $\otimes: \mathscr{C} \times \mathscr{C} \longrightarrow \mathscr{C}$ and consider, as above, a ring object $A$ in $\mathscr{C}$ with associative and unital multiplication $\mu: A \otimes A \rightarrow A$ (details are recalled in Section 2). The $A$-modules in $\mathscr{C}$ are simply objects $x$ in $\mathscr{C}$ together with an $A$-action $A \otimes x \rightarrow x$ satisfying the usual rules. We can form the category $A-\operatorname{Mod}_{\mathscr{C}}$ of $A$-modules in $\mathscr{C}$ and we have an extension-of-scalars functor $F_{A}: \mathscr{C} \rightarrow A-\operatorname{Mod}_{\mathscr{C}}$, which maps $y$ to $A \otimes y$, as one would expect. As in commutative algebra, the ring object $A$ is said to be separable if $\mu$ admits a section $\sigma: A \rightarrow A \otimes A$ which is $A$-linear on both sides. A very special example of separability occurs if $\mu$ is an isomorphism (with inverse $\sigma$ ) 
in which case the extension-of-scalars $\mathscr{C} \rightarrow A-\operatorname{Mod}_{\mathscr{C}}$ is just a localization of $\mathscr{C}$. But general separable extensions are more flexible than localizations. For instance, in algebraic geometry they include finite étale extensions of affine schemes by Balmer [1, Corollary 6.6].

Separable extensions are particularly nice for another reason, beyond the analogy with the étale topology, namely, they can be performed on triangulated categories without resorting to models; see [1]. Since all the above categories $\mathscr{C}(G)$ are triangulated, our results establish a connection between these equivariant theories and the "tensortriangular geometry" of étale extensions, as initiated in Balmer [2].

Understanding restriction as an étale extension has already found applications in modular representation theory (see [3, Part II]) and it is legitimate to expect similar developments in our new examples. This will be the subject of further work.

Let us say a word about our hypothesis that $G / H$ is finite. In Section 6 , we prove the following result which shows that Theorem 1.1 cannot hold without some finiteness assumption on $G / H$ :

1.5 Theorem Let $G$ be a connected compact Lie group and let $H \leq G$ be a nontrivial finite subgroup. Then the right adjoint to the restriction functor $\operatorname{Res}_{H}^{G}: \operatorname{SH}(G) \rightarrow$ $\mathrm{SH}(H)$ is not faithful. In particular, $\operatorname{Res}_{H}^{G}$ is not an extension-of-scalars.

Let us explain what is going on. The proofs of Theorems 1.1-1.3 all follow a similar pattern that we isolate in the preparatory Section 2. In technical terms, we prove separable monadicity of the standard restriction-coinduction adjunction and then show that the monad associated to this adjunction is given by a separable ring object. In an ideal world, for a general subgroup $H$ of a general group $G$, we would expect the first property (monadicity) to hold when $G / H$ is discrete and the second (the ring object) when $G / H$ is compact. Then our hypothesis that $G / H$ is finite would simply result from assuming simultaneously that $G / H$ is discrete and compact. That would be the ideal treatment. However, things turn out to be more complicated, mostly due to the current state-of-development of our examples.

Firstly, $G$-equivariant stable homotopy theory is simply not developed for non-compact groups. Similarly, $G$-equivariant KK-theory, although defined for locally compact groups, lacks enough adjoints if we do not assume $G / H$ compact (see the technical reasons in Remark 4.4). These restrictions prevent a uniform treatment beyond the case of $G / H$ finite. Trying to lift those restrictions would be a massive undertaking, going way beyond the goal of the present paper. We found our results diverse enough as they 
are, without trying to push them into unnecessary complications. For instance, Theorems 1.1-1.3 are already interesting for finite groups, where they hold unconditionally. For the same reasons, we renounced treating the $G$-equivariant derived category over nondiscrete groups, à la Bernstein and Lunts [4].

Our goal is to show that restriction to a subgroup can be understood as an étale extension in a broad range of settings beyond representation theory. The above sample should provide convincing evidence of this ubiquity and should encourage our readers to try proving similar results for their favorite equivariant categories. It is likely that future investigations will produce further examples of this phenomenon and we are confident that the method of proof presented in Section 2 will be useful for such generalizations.

Acknowledgements P B was supported by NSF grant number DMS-1303073. ID'A was partially supported by the Labex CEMPI (ANR-11-LABX-0007-01).

\section{General approach}

\section{Separable monadicity}

Let us briefly recall some standard facts about monads and separability; we refer the reader to [1] and [3] for further details. A monad on a category $\mathscr{C}$ consists of an endofunctor $\mathbb{A}: \mathscr{C} \rightarrow \mathscr{C}$ equipped with natural transformations $\mu: \mathbb{A} \circ \mathbb{A} \rightarrow \mathbb{A}$ and $\eta: \operatorname{Id}_{\mathscr{C}} \rightarrow \mathbb{A}$ such that $\mu$ is an associative multiplication $(\mu \circ \mathbb{A} \mu=\mu \circ \mu \mathbb{A})$ for which $\eta$ is a two-sided identity $(\mu \circ \mathbb{A} \eta=\mathrm{id}=\mu \circ \eta \mathbb{A})$. An $\mathbb{A}$-module in $\mathscr{C}$ consists of a pair $(x, \rho)$ where $x$ is an object of $\mathscr{C}$ and $\rho: \mathbb{A} x \rightarrow x$ is a morphism (the "action" of $\mathbb{A}$ on $x$ ) making the evident associativity and unit diagrams commute in $\mathscr{C}$. A morphism of $\mathbb{A}$-modules $(x, \rho)$ and $\left(x^{\prime}, \rho^{\prime}\right)$ is a morphism $f: x \rightarrow x^{\prime}$ in $\mathscr{C}$ commuting with the actions. We denote by $\mathbb{A}-\operatorname{Mod}_{\mathscr{C}}$ the resulting category of modules, which is part of the Eilenberg-Moore adjunction $F_{\mathbb{A}}: \mathscr{C} \rightleftarrows \mathbb{A}-\operatorname{Mod}_{\mathscr{C}}: U_{\mathbb{A}}$. The left adjoint $F_{\mathbb{A}}$ sends an object $c \in \mathscr{C}$ to the free $\mathbb{A}$-module $F_{\mathbb{A}}(c):=\left(\mathbb{A} c, \mu_{c}: \mathbb{A} \mathbb{A} c \rightarrow \mathbb{A} c\right)$, and the right adjoint sends a module $(x, \rho)$ to its underlying object $U_{\mathbb{A}}(x, \rho):=x$.

Any adjunction $F: \mathscr{C} \rightleftarrows \mathscr{D}: U$ with unit $\eta: \operatorname{Id}_{\mathscr{C}} \rightarrow U F$ and counit $\epsilon: F U \rightarrow \operatorname{Id}_{\mathscr{D}}$ defines a monad $\mathbb{A}=(U F, U \epsilon F, \eta)$ on $\mathscr{C}$ and we can consider the Eilenberg-Moore adjunction associated with this monad as above. There is a unique "comparison" functor $E: \mathscr{D} \rightarrow \mathbb{A}-\operatorname{Mod}_{\mathscr{C}}$ such that $E \circ F=F_{\mathbb{A}}$ and $U_{\mathbb{A}} \circ E=U$

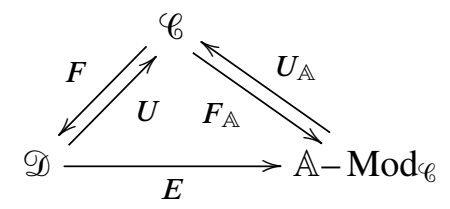


and we say that the adjunction $F \dashv U$ is monadic if the comparison functor $E$ : $\mathscr{D} \rightarrow$ $\mathbb{A}-\operatorname{Mod}_{\mathscr{C}}$ is an equivalence of categories. Concretely, $E$ is given by

$$
E(d)=\left(U d, U \epsilon_{d}: U F U d \rightarrow U d\right)
$$

on objects $d \in \mathscr{D}$ and by $U(f)$ on morphisms $f: d \rightarrow d^{\prime}$.

We stress that, although the construction of $E$ is formal, the property that it is an equivalence is highly nontrivial and simply fails in general. At the extreme, taking $\mathscr{D}$ arbitrary and $\mathscr{C}=0$ (hence $\mathbb{A}-\operatorname{Mod}_{\mathscr{C}}=0$ ) shows that $E$ can get as bad as one wants. Hence, monadicity is a nontrivial property. Note that since $U_{\mathbb{A}}$ is faithful, a necessary condition for $E$ to be an equivalence is faithfulness of $U$.

A monad $\mathbb{A}: \mathscr{C} \rightarrow \mathscr{C}$ is said to be separable if the multiplication $\mu: \mathbb{A} \circ \mathbb{A} \rightarrow \mathbb{A}$ admits a natural section $\sigma: \mathbb{A} \rightarrow \mathbb{A} \circ \mathbb{A}$ which is $\mathbb{A}, \mathbb{A}$-linear: $\mu \mathbb{A} \circ \mathbb{A} \sigma=\sigma \circ \mu=\mathbb{A} \mu \circ \sigma \mathbb{A}$.

2.2 Lemma Let $F: \mathscr{C} \leftrightarrows \mathscr{D}: U$ be an adjunction between idempotent-complete additive categories, and assume that the counit $\epsilon: F U \rightarrow \mathrm{Id}_{\mathscr{D}}$ admits a section, ie, a natural morphism $\xi: \mathrm{Id}_{\mathscr{D}} \rightarrow F U$ such that $\epsilon \circ \xi=\mathrm{id}$. Then the adjunction is separably monadic. That is, the monad $U F$ on $\mathscr{b}$ is separable and the Eilenberg-Moore comparison functor $E$ in (2.1) is an equivalence. A quasi-inverse $E^{-1}: \mathbb{A}-\operatorname{Mod}_{\mathscr{C}} \stackrel{\sim}{\longrightarrow} \mathscr{D}$ is obtained by sending $(x, \rho) \in \mathbb{A}-\operatorname{Mod}_{\mathscr{C}}$ to the image $E^{-1}(x, \rho):=\operatorname{Im}(e)$ of the idempotent $e^{2}=e:=F(\rho) \circ \xi_{F(x)}$ on $F(x) \in \mathscr{D}$.

Proof The fact that $E$ is an equivalence is [3, Lemma 2.10]. In order to show that the described $E^{-1}$ is quasi-inverse to $E$, it suffices to show that it is a welldefined functor and that $E^{-1} E \cong \operatorname{Id}_{\mathscr{D}}$. The latter is a straightforward verification. For the former, it suffices to show that $e=F(\rho) \circ \xi_{F(x)}$ is idempotent; then its image exists because $\mathscr{C}$ is idempotent-complete, and the assignment $(x, \rho) \mapsto \operatorname{Im}(e)$ extends to a well-defined functor in the evident way, by sending $f:(x, \rho) \rightarrow\left(x^{\prime}, \rho^{\prime}\right)$ to $e^{\prime} F(f) e: \operatorname{Im}(e) \rightarrow \operatorname{Im}\left(e^{\prime}\right)$. To see why $e^{2}=e$, consider the following diagram:

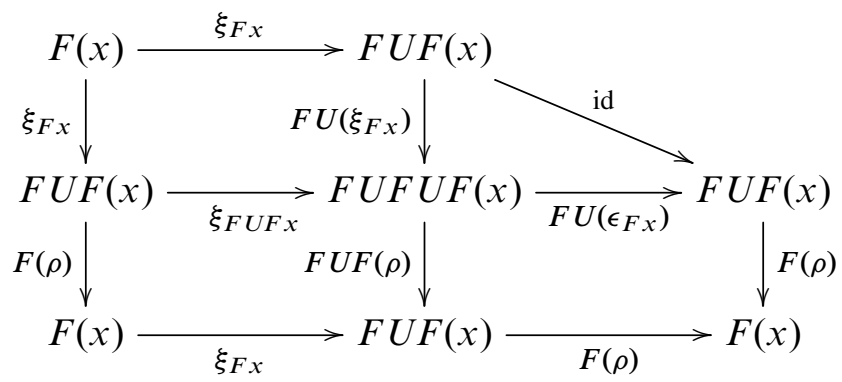

The two left squares commute by naturality of $\xi$, the right square because $\rho$ is an action and the triangle because $\xi$ is a section of $\epsilon$. The perimeter reads $e^{2}=e$. 
2.3 Example Assume that $(\mathscr{C}, \otimes, \mathbb{1})$ is a tensor category, by which we mean a symmetric monoidal category with tensor $\otimes$ and unit object $\mathbb{1}$. Let $A=(A, \mu, \iota)$ be a ring object (aka monoid) in $\mathscr{C}$, that is, an object $A \in \mathscr{C}$ equipped with a multiplication $\mu: A \otimes A \rightarrow A$ and unit $\iota: \mathbb{1} \rightarrow A$ such that the associativity axiom $\mu\left(\mu \otimes \operatorname{id}_{A}\right)=$ $\mu\left(\operatorname{id}_{A} \otimes \mu\right)$ and unit axioms $\mu\left(\iota \otimes \mathrm{id}_{A}\right)=\mathrm{id}_{A}=\mu\left(\operatorname{id}_{A} \otimes \iota\right)$ hold in $\mathscr{C}$. Then $A$ defines a monad $\mathbb{A}=A \otimes-: \mathscr{C} \rightarrow \mathscr{C}$ with multiplication $\mu \otimes-$ and unit $\iota \otimes-$ (adjusted by the associativity and unit constraints of $\otimes$ ). In this case, we use the notation

$$
F_{A}: \mathscr{C} \leftrightarrows A-\operatorname{Mod}_{\mathscr{C}}: U_{A}
$$

for the resulting Eilenberg-Moore adjunction and call $F_{A}$ the extension-of-scalars functor, as in Section 1. Thus an $A$-module $(x, \rho) \in A-\operatorname{Mod}_{\mathscr{C}}$ consists of an object $x \in \mathscr{C}$ equipped with a map $\rho: A \otimes x \rightarrow x$ such that $\rho\left(\mu \otimes \operatorname{id}_{x}\right)=\rho\left(\operatorname{id}_{A} \otimes \rho\right)$ and $\rho\left(\iota \otimes \mathrm{id}_{x}\right)=\mathrm{id}_{x}$ in $\mathscr{C}$. If the multiplication $\mu: A \otimes A \rightarrow A$ admits an $A, A$-linear section $\sigma: A \rightarrow A \otimes A$ then $A$ is said to be separable. In this case the associated monad $A \otimes-$ will be a separable monad.

\section{The projection formula}

Assume that both $\mathscr{C}$ and $\mathscr{D}$ are tensor categories and that $F: \mathscr{C} \rightarrow \mathscr{D}$ is a tensor functor ( = strong symmetric monoidal functor), ie it comes with coherent isomorphisms $\mathbb{1} \stackrel{\sim}{\longrightarrow} F(\mathbb{1})$ and $F(x) \otimes F(y) \stackrel{\sim}{\longrightarrow} F(x \otimes y)$. A right adjoint $U$ of $F$ inherits the structure of a lax tensor functor, consisting of coherent maps $\iota: \mathbb{1} \rightarrow U(\mathbb{1})$ and $\lambda: U(x) \otimes U(y) \rightarrow$ $U(x \otimes y)$. They are defined by

$$
\iota: \mathbb{1} \stackrel{\eta}{\longrightarrow} U F(\mathbb{1}) \stackrel{\sim}{\longrightarrow} U(\mathbb{1}),
$$

(2.4) $\lambda: U(x) \otimes U(y) \stackrel{\eta}{\longrightarrow} U F(U x \otimes U y) \stackrel{\sim}{\longrightarrow} U(F U x \otimes F U y) \stackrel{U(\epsilon \otimes \epsilon)}{\longrightarrow} U(x \otimes y)$,

and they are not necessarily invertible. Lax monoidal functors preserve ring objects. In particular, we obtain a commutative ring object $A:=(U(\mathbb{1}), \mu, \iota)$ in $\mathscr{b}$, where we endow $U(\mathbb{1})$ with the unit map $\iota$ as above and the multiplication

$$
\mu: U(\mathbb{1}) \otimes U(\mathbb{1}) \stackrel{\lambda}{\longrightarrow} U(\mathbb{1} \otimes \mathbb{1}) \stackrel{\sim}{\longrightarrow} U(\mathbb{1}) .
$$

The lax monoidal structure of $U$ also defines a natural transformation

$$
\pi: U(y) \otimes x \stackrel{\mathrm{id} \otimes \eta}{\longrightarrow} U(y) \otimes U F(x) \stackrel{\lambda}{\longrightarrow} U(y \otimes F(x))
$$

for all $x \in \mathscr{C}$ and $y \in \mathscr{D}$, which we call the projection morphism.

2.7 Definition We say that the projection formula holds for the adjunction $F \dashv U$ when the natural morphism $\pi: U(x) \otimes y \rightarrow U(x \otimes F(y))$ of (2.6) is an isomorphism for all $x \in \mathscr{C}$ and $y \in \mathscr{D}$. 
Even when the morphism $\pi$ is not an isomorphism, it compares two monads on $\mathscr{b}$ : the monad $A \otimes(-)$ induced by the ring object $A=(U \mathbb{1}, \mu, \iota)$ and the $\operatorname{monad} \mathbb{A}=U F$ induced by the adjunction. We now prove that the natural transformation $\pi$ is always compatible with those additional structures:

2.8 Lemma With the above notation, the natural map

$$
\pi: U(\mathbb{1}) \otimes x \rightarrow U(\mathbb{1} \otimes F x) \cong U F(x)
$$

is a morphism $A \otimes(-) \rightarrow \mathbb{A}$ of monads on $\mathfrak{b}$.

Proof We must verify that $\pi$ identifies the units and multiplications of the two monads. Concretely, we must show that the following diagrams commute in $\mathscr{b}$ :
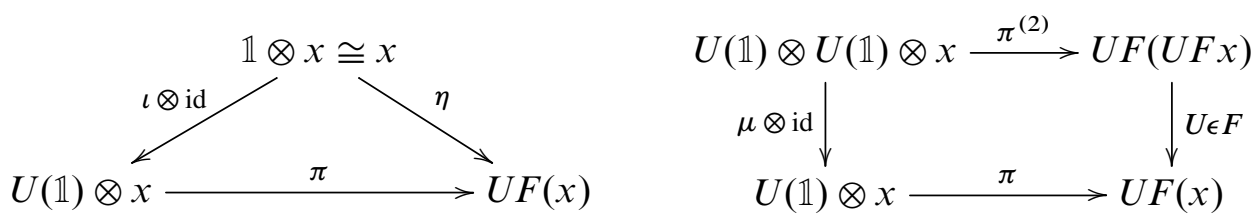

Here $\pi^{(2)}:=\left(\pi_{U F x}\right)\left(\mathrm{id} \otimes \pi_{x}\right)=\left(\mathrm{id} \otimes \pi_{x}\right)\left(\pi_{U(\mathbb{1}) \otimes x}\right)$ denotes the two-fold application of $\pi$. The commutativity of the above triangle follows from that of the diagram

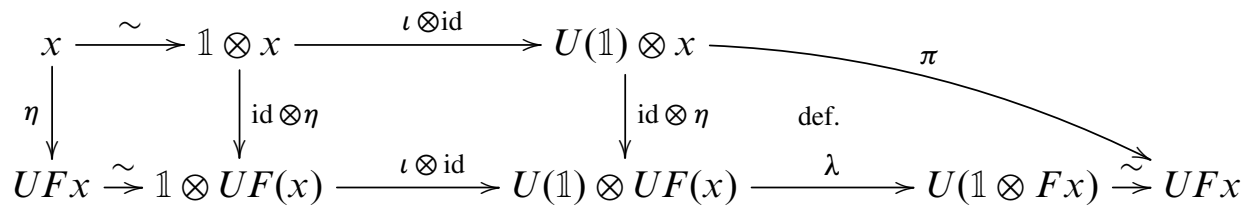

once we note that the bottom row is the identity; the latter holds because the following diagram commutes (since $U$ is lax monoidal)

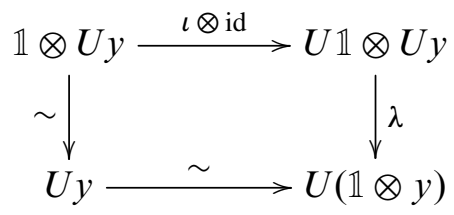

for all $y($ plug $y:=F x)$. Next we check the commutativity of the diagram

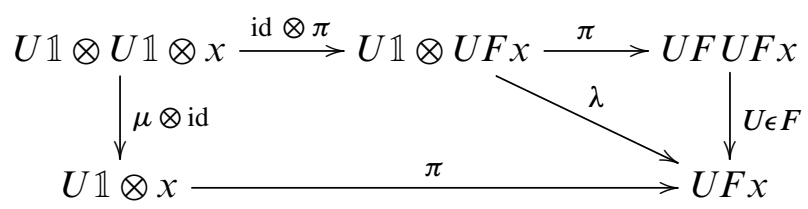


by using the definition of $\pi=\lambda$ (id $\otimes \eta$ ) in (2.6), commutativity of the diagram

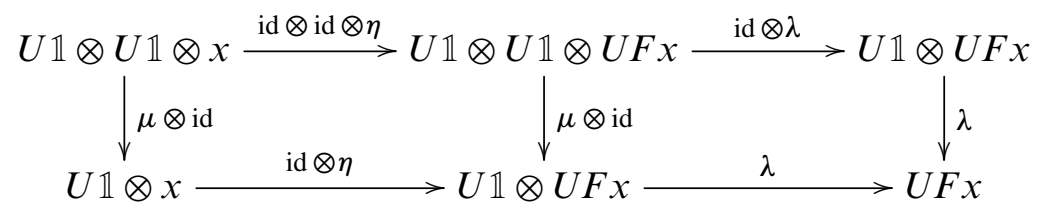

which reads $\pi(\mu \otimes \mathrm{id})=\lambda(\mathrm{id} \otimes \pi)$, and commutativity of the diagram

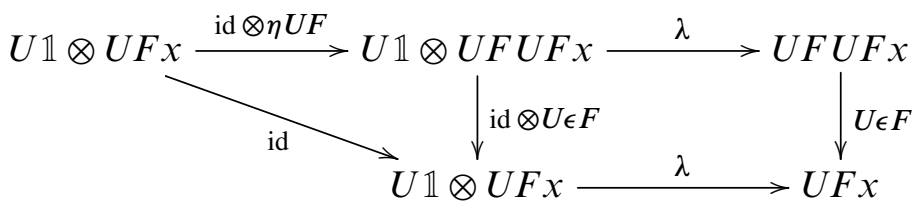

which reads $\lambda=U \epsilon F \pi$. We have suppressed unital isomorphisms for readability.

We now prove an analogue of Beck monadicity in our framework:

2.9 Theorem Let $F: \mathscr{C} \leftrightarrows \mathscr{D}: U$ be an adjunction of idempotent-complete additive tensor categories, where $F$ is a tensor functor. Assume moreover that:

(a) The counit of the adjunction $\epsilon: F U \rightarrow \mathrm{Id}_{\mathscr{D}}$ admits a natural section.

(b) The projection formula holds $U(x) \otimes y \cong U(x \otimes F(y))$; see Definition 2.7.

Then the adjunction is monadic and the associated monad is isomorphic to the one induced by the commutative ring object $A=(U(\mathbb{1}), \mu, \iota)$ in $\mathscr{b}$; see (2.5). Thus there is a (unique) equivalence $E: \mathscr{D} \stackrel{\sim}{\longrightarrow} A-\operatorname{Mod}_{\mathscr{C}}$ identifying the given adjunction $F \dashv U$ with the free-forgetful adjunction $F_{A} \dashv U_{A}$, up to isomorphism; see (2.1).

Proof Just combine Lemmas 2.2 and 2.8. Explicitly, the equivalence $E: \mathscr{D} \stackrel{\sim}{\longrightarrow}$ $U(\mathbb{1})-\operatorname{Mod}_{\mathscr{C}}$ sends $d \in \mathscr{D}$ to the $U(\mathbb{1})$-module $E(d):=U(d)$ with action given by

$$
U(\mathbb{1}) \otimes U(d) \stackrel{\lambda}{\longrightarrow} U(\mathbb{1} \otimes d) \simeq U d .
$$

Recall now the situation of Section 1. In each of the three examples discussed there, we are given suitable groups $G$ and $H$, where $H$ is a subgroup of finite index in $G$. We have associated tensor triangulated categories $\mathscr{C}:=\mathscr{C}(G)$ and $\mathscr{D}:=\mathscr{C}(H)$ and a tensor exact restriction functor $F:=\operatorname{Res}_{H}^{G}: \mathscr{C}(G) \rightarrow \mathscr{C}(H)$ admitting a right adjoint $U:=\operatorname{CoInd}_{H}^{G}: \mathscr{C}(H) \rightarrow \mathscr{C}(G)$. In the remaining three sections, we are going to deduce Theorems 1.1, 1.2 and 1.3 from Theorem 2.9, by verifying in each case that the hypotheses (a) and (b) hold. We will sometimes abbreviate the corresponding monad as $\mathbb{A}=\mathbb{A}_{H}^{G}:=\operatorname{CoInd}_{H}^{G} \circ \operatorname{Res}_{H}^{G}$. 
2.10 Remark Although the splitting of the counit implies (see Lemma 2.2) that the monad $U F \simeq U(\mathbb{1}) \otimes(-)$ is separable, it does not follow a priori that the ring object $U(\mathbb{1})$ is itself separable. However, in all examples, the ring object $A=A_{H}^{G} \simeq U(\mathbb{1})$ will be separable with an obvious $A, A$-linear section $\sigma: A_{H}^{G} \rightarrow A_{H}^{G} \otimes A_{H}^{G}$ of its multiplication, which will always be induced by the diagonal map $G / H \rightarrow G / H \times G / H$. This is clearly the simplest explanation for separability of $U(\mathbb{1})$ in the examples. However, one could also expand the above abstract treatment to obtain separability of $U(\mathbb{1})$ from general arguments. Indeed, in all our examples, the functor $F$ also has a left adjoint $L$ which is isomorphic to $U$ and the section $x \rightarrow F U(x)$ we construct of the counit of $F \dashv U$ coincides with the unit $x \rightarrow F L(x)$ of the $L \dashv F$ adjunction under this isomorphism $L \simeq U$. Using compatibility of left and right projection formulas, one can then show that $U(\mathbb{1})$ is indeed separable in that case. Further details are left to the interested reader.

\section{Equivariant stable homotopy theory}

Let $G$ be a compact Lie group and let $\mathrm{SH}(G)$ denote the stable homotopy category of (genuine) $G$-spectra, in the sense of [10]. This is a compactly generated tensor triangulated category with the smash product of $G$-spectra, $-\wedge-$, and unit $S=\Sigma^{\infty} S^{0}$. For any closed subgroup $H \leq G$, we have a restriction tensor functor $\operatorname{Res}_{H}^{G}: \operatorname{SH}(G) \rightarrow$ $\mathrm{SH}(H)$ which admits a left adjoint, induction, denoted $G_{+} \wedge_{H}-$, and a right adjoint, coinduction, denoted $F_{H}\left(G_{+},-\right)$. The two adjoints are related by the Wirthmüller isomorphism, which is a natural isomorphism

$$
\omega_{X}: F_{H}\left(G_{+}, X\right) \stackrel{\sim}{\longrightarrow} G_{+} \wedge_{H}\left(X \wedge S^{-L}\right),
$$

defined for any $H$-spectrum $X$, where $L$ is the tangent $H$-representation of $G / H$ at the identity coset $e H$. (See [11, Section XVI.4] and [12].) Note that if $H$ has finite index in $G$ then $L=0$ and in this case $\omega_{X}$ provides an isomorphism between the induction and coinduction functors: $F_{H}\left(G_{+}, X\right) \stackrel{\sim}{\longrightarrow} G_{+} \wedge_{H} X$.

3.2 Lemma The restriction-coinduction adjunction

$$
\operatorname{Res}_{H}^{G}: \mathrm{SH}(G) \leftrightarrows \mathrm{SH}(H): F_{H}\left(G_{+},-\right)
$$

between the equivariant stable homotopy categories satisfies the projection formula (Definition 2.7).

Proof As noted in [12, Remark 1.2], this follows from the Wirthmüller isomorphism. We briefly recall the argument. By [6, Proposition 3.2] the projection map

$$
\pi: Y \wedge F_{H}\left(G_{+}, X\right) \longrightarrow F_{H}\left(G_{+}, \operatorname{Res}_{H}^{G}(Y) \wedge X\right)
$$


as in (2.6) is invertible whenever $X$ is a dualizable (ie compact) object of $\mathrm{SH}(H)$. Smashing with any object preserves coproducts. Hence so does $F_{H}\left(G_{+},-\right)$, by the Wirthmüller isomorphism (3.1). Thus the two functors (in $X$ ) that are compared by $\pi$ are both exact and commute with coproducts. Since $\mathrm{SH}(H)$ is compactly generated, it follows that $\pi$ is invertible for arbitrary $X$ as well.

3.3 Lemma Let $G$ be a compact Lie group and let $H \leq G$ be a closed subgroup of finite index in $G$. Then the counit of the restriction-coinduction adjunction between $\mathrm{SH}(G)$ and $\mathrm{SH}(H)$ has a natural section.

Proof Since $G / H$ is finite, hence discrete, the $H$-space $G_{+}$decomposes as a coproduct $(G-H) \sqcup H_{+}$and we can define for any based $H$-space $X$ a continuous $H$-equivariant map $\xi_{X}: X \rightarrow F_{H}\left(G_{+}, X\right)$ by

$$
\xi_{X}(x)(g)= \begin{cases}g x & \text { if } g \in H, \\ * & \text { if } g \notin H .\end{cases}
$$

This map is natural in $X$ and defines a section of the counit

$$
\epsilon_{X}: F_{H}\left(G_{+}, X\right) \longrightarrow X, \quad \varphi \longmapsto \varphi(1)
$$

of the space-level restriction-coinduction adjunction. At the level of spectra, recall from [10, Section II.4, page 77] that coinduction is defined spacewise without the need to spectrify; that is, for an $H$-spectrum $D$, the $G-\operatorname{spectrum} F_{H}\left(G_{+}, D\right)$ is defined for every $G$-representation $V$ in the indexing universe by $F_{H}\left(G_{+}, D\right)(V):=$ $F_{H}\left(G_{+}, D(V)\right)$ where the right-hand side is the space-level coinduction functor. One checks that the maps $\xi_{D(V)}$ define a map of $H$-spectra $D \rightarrow F_{H}\left(G_{+}, D\right)$ by using the definition of the structure maps of $F_{H}\left(G_{+}, D\right)$ and the commutativity of

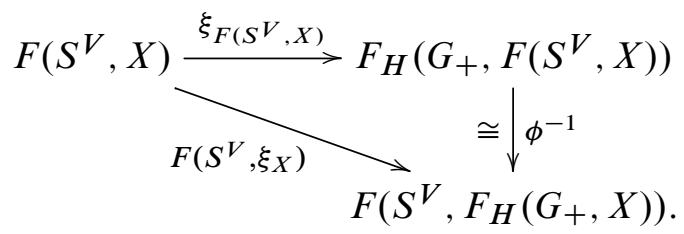

Here $\phi^{-1}$ is the $G$-homeomorphism defined on page 76 of [10] and the commutativity of diagram (3.4) follows immediately from the definitions. In this way, we have constructed a section of the counit of the restriction-coinduction adjunction between the categories of $G$-spectra and $H$-spectra. Coinduction preserves weak equivalences so this splitting passes without difficulty to a splitting of the counit of the adjunction between homotopy categories: $\mathrm{SH}(G) \rightleftarrows \mathrm{SH}(H)$. 
Proof of Theorem 1.1. The theorem follows from Theorem 2.9, since its hypotheses (a) and (b) have been verified in Lemmas 3.3 and 3.2. It remains only to describe the ring object $A=(A, \mu, \iota)$ in $\operatorname{SH}(G)$. By definition, we have $A=F_{H}\left(G_{+}, S\right)$ and the Wirthmüller isomorphism identifies it with the $G$-equivariant suspension spectrum $G_{+} \wedge_{H} S \cong \Sigma^{\infty}(G / H)_{+}$. Under this isomorphism, the multiplication in $F_{H}\left(G_{+}, S\right)$ becomes the multiplication $A_{H}^{G} \otimes A_{H}^{G} \rightarrow A_{H}^{G}$ announced in the Introduction. Under the isomorphism $\Sigma^{\infty}(G / H)_{+} \wedge \Sigma^{\infty}(G / H)_{+} \cong \Sigma^{\infty}(G / H \times G / H)_{+}$it is given by the map $\left(\gamma, \gamma^{\prime}\right) \mapsto \gamma$ when $\gamma=\gamma^{\prime}$ and $*$ otherwise; see (1.4). An obvious section $\sigma$ is given by $\gamma \mapsto(\gamma, \gamma)$, showing that $A_{H}^{G}$ is indeed separable as a ring object. See Remark 2.10.

\section{Equivariant KK-theory}

We begin with some recollections on equivariant KK-theory. Details can be found in [14] and the references therein.

Let $G$ be a second countable locally compact Hausdorff group. For short, we use the term $G$-algebra to mean a (topologically) separable ${ }^{1}$ complex $\mathrm{C}^{*}$-algebra equipped with a continuous left $G$-action $G \times A \rightarrow A$ by $*$-isomorphisms. We denote by $\operatorname{Alg}(G)$ the category of $G$-algebras and $G$-equivariant $*$-homomorphisms (morphisms for short). It is a symmetric monoidal category when equipped with the minimal tensor product $-\otimes-$ (ie, the completion of the algebraic tensor product $-\otimes_{\mathbb{C}}-$ with respect to the minimal $\mathrm{C}^{*}$-norm), where $G$ acts diagonally on tensor products: $g(a \otimes b):=g a \otimes g b$.

The $G$-equivariant Kasparov category, here denoted $\operatorname{KK}(G)$, has the same objects as $\operatorname{Alg}(G)$ and its Hom sets are Kasparov's $G$-equivariant bivariant K-theory groups $\operatorname{Hom}_{\mathrm{KK}(G)}(A, B)=K K^{G}(A, B)$ with composition given by the so-called Kasparov product. It is a tensor triangulated category admitting all countable coproducts, so in particular it is an idempotent-complete additive category. It comes equipped with a canonical tensor functor $\operatorname{Alg}(G) \rightarrow \mathrm{KK}(G)$ which is the identity on objects. We will not distinguish notationally between a morphism of $\operatorname{Alg}(G)$ and its canonical image in $\operatorname{KK}(G)$.

4.1 Construction Let $H$ be a closed subgroup of $G$ and assume already, for simplicity, that the quotient $G / H$ is a finite discrete space (see Remark 4.4 for more general results). By restricting $G$-actions to $H$, we obtain a restriction functor

${ }^{1} \mathrm{~A} \mathrm{C} \mathrm{C}^{*}$-algebra is separable if it admits a countable subset which is dense (for the norm topology). This is not related to the separability of monads and rings discussed in Section 2. 
$\operatorname{Res}_{H}^{G}: \operatorname{Alg}(G) \rightarrow \operatorname{Alg}(H)$. Define coinduction $\operatorname{CoInd}_{H}^{G}: \operatorname{Alg}(H) \rightarrow \operatorname{Alg}(G)$ as follows. Its value on an $H$-algebra $B$ is the $G$-algebra

$$
\operatorname{CoInd}_{H}^{G}(B):=\mathrm{C}_{b}(G, B)^{H}
$$

of those bounded continuous functions $f: G \rightarrow B$ that are $H$-invariant: $f(h x)=$ $h f(x)$ for all $h \in H$ and $x \in G$. This is again a separable $C^{*}$-algebra with the supremum norm and the pointwise algebraic operations. The left $G$-action is given by $(g \cdot f)(x):=f(x g)$. The functoriality is obtained by composing functions with morphisms $B \rightarrow B^{\prime}$. For all $A \in \operatorname{Alg}(G)$ and $B \in \operatorname{Alg}(H)$, we define the unit and counit natural transformations by the "usual" formulas

$$
\begin{aligned}
& \eta_{A}: A \rightarrow \operatorname{CoInd}_{H}^{G} \operatorname{Res}_{H}^{G}(A), \quad a \mapsto \eta_{A}(a):=(G \ni t \mapsto t a \in A) \\
& \epsilon_{B}: \operatorname{Res}_{H}^{G} \operatorname{CoInd}_{H}^{G}(B) \rightarrow B, \quad f \mapsto \epsilon_{B}(f):=f(1)
\end{aligned}
$$

for $a \in A$ and $f \in \operatorname{CoInd}_{H}^{G}(B)$.

\subsection{Lemma The functor}

$$
\operatorname{Res}_{H}^{G}: \operatorname{Alg}(G) \rightarrow \operatorname{Alg}(H)
$$

is left adjoint to

$$
\operatorname{CoInd}_{H}^{G}: \operatorname{Alg}(H) \rightarrow \operatorname{Alg}(G),
$$

with the above $\eta$ and $\epsilon$ as unit and counit. Moreover:

(a) The counit $\epsilon$ admits a natural section.

(b) The adjunction satisfies the projection formula (Definition 2.7).

Proof This adjunction is well-known (see [16, page 231] or [15, Proposition 38]) and works equally fine with $G / H$ compact, although we could not locate the explicit unit and counit in the literature. The verifications that the above $\eta$ and $\epsilon$ are well-defined and satisfy the triangle equalities are immediate.

Since $G / H$ is assumed discrete, for each $b \in B$ the formula

$$
G \ni t \mapsto \xi_{B}(b)(t):= \begin{cases}t b & \text { if } t \in H, \\ 0 & \text { if } t \notin H,\end{cases}
$$

yields a well-defined map $\xi_{B}(b) \in \mathrm{C}_{b}(G, B)^{H}$. Moreover, the assignment $b \mapsto \xi_{B}(b)$ defines an $H$-equivariant morphism $\xi_{B}: B \rightarrow \mathrm{C}_{b}(G, B)^{H}$ and therefore a natural transformation $\xi: \operatorname{Id}_{\mathrm{Alg}(H)} \rightarrow \operatorname{Res}_{H}^{G} \operatorname{CoInd}_{H}^{G}$. Since $\epsilon_{B} \xi_{B}(b)=\xi_{B}(b)(1)=1 b=b$ for all $b \in B$, we see that $\xi$ provides the natural section claimed in part (a). 
Let us prove part (b). By unfolding the definitions, we see that the present incarnation of the projection map (2.6) is the following morphism of $G$-algebras,

$$
\begin{aligned}
\pi: \quad \operatorname{CoInd}_{H}^{G}(B) \otimes A & \longrightarrow \operatorname{CoInd}_{H}^{G}\left(B \otimes \operatorname{Res}_{H}^{G}(A)\right), \\
f \otimes a & \longmapsto \pi(f \otimes a)=(G \ni t \mapsto f(t) \otimes t a),
\end{aligned}
$$

for all $B \in \operatorname{Alg}(H)$ and $A \in \operatorname{Alg}(G)$. The above formula defines $\pi$ on simple algebraic tensors, and extends uniquely to the minimal tensor product by linearity and continuity. Once again, the fact that this is an isomorphism is well-known to the experts but the details are hard to find in the literature. For $G / H$ finite it is actually easy. Choose a full set $R \subset G$ of representatives modulo $H$. Then, forgetting actions, the inclusion $R \hookrightarrow G$ induces a natural (!) isomorphism of (nonequivariant) $\mathrm{C}^{*}$-algebras $\rho: \operatorname{CoInd}_{H}^{G}(D) \stackrel{\sim}{\longrightarrow} \prod_{r \in R} D$ for all $D \in \operatorname{Alg}(H)$, by $\rho(f)=(f(r))_{r}$. We thus obtain the following commutative square of $\mathrm{C}^{*}$-algebras

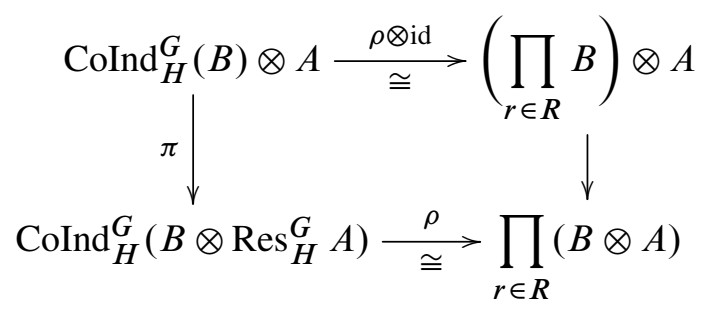

where the right vertical map is defined, on simple tensors, by $\left(b_{r}\right)_{r} \otimes a \mapsto\left(b_{r} \otimes r a\right)_{r}$. The latter is invertible because $R$ is finite, hence so is $\pi$.

4.4 Remark If $G / H$ is compact, but not necessarily discrete, Construction 4.1 still works verbatim to provide the right adjoint of restriction, but some nontrivial analysis (using that $H$ acts properly on $G$ ) is needed to prove that the functor yields separable $\mathrm{C}^{*}$-algebras and satisfies the projection formula. When $H$ is any closed subgroup, without any hypothesis on $G / H$, it is convenient to consider an "induction" functor $\operatorname{Ind}_{H}^{G}: \operatorname{Alg}(H) \rightarrow \operatorname{Alg}(G)$ defined by the subalgebra

$$
\operatorname{Ind}_{H}^{G}(B):=\left\{f \in \mathrm{C}_{b}(G, B)^{H} \mid(t \mapsto\|f(t)\|) \in \mathrm{C}_{0}(G / H)\right\}
$$

of $\operatorname{CoInd}_{H}^{G}(B)$. The same analytical arguments apply to show that $\operatorname{Ind}_{H}^{G}$ takes separable values and satisfies the projection formula, in full generality. Clearly $\operatorname{Ind}_{H}^{G}=\operatorname{CoInd}_{H}^{G}$ when $G / H$ is compact, so it is right adjoint to $\operatorname{Res}_{H}^{G}$ in this case. If instead $G / H$ is discrete then $\operatorname{Ind}_{H}^{G}$ becomes, at the level of the Kasparov categories, left adjoint to restriction, thus earning its name; see [15, Section 2.6]. It is not known whether there exists, unconditionally, a left or right adjoint to restriction on the Kasparov categories. In our view, this anomalous behavior - quite unlike the situation in representation theory 
or equivariant stable homotopy - is a cost of the endemic countability hypotheses required by the analytical constructions traditionally involved with KK-theory, which prevent the Kasparov categories from admitting arbitrary small coproducts.

Proof of Theorem 1.2 We are assuming that $G / H$ is finite, hence discrete, so we can apply Lemma 4.2. We claim that the conclusions of Lemma 4.2 still hold at the level of KK-theory, not only at the level of algebras and algebra morphisms.

Indeed, recall that the canonical functor $\operatorname{Alg}(G) \rightarrow \operatorname{Alg}(G)\left[W_{G}^{-1}\right]=\operatorname{KK}(G)$ is a localization of categories, obtained by inverting precisely the set $W_{G}$ of $G$-equivariant $K K$-equivalences, and similarly for $H$. This follows immediately from Meyer's universal property of equivariant KK-theory [13], and from the following easy (and wellknown) observation: each of the three properties of an additive functor $F: \operatorname{Alg}(G) \rightarrow \mathscr{C}$ for which $\operatorname{KK}(G)$ is universal - namely, homotopy invariance, $\mathrm{C}^{*}$-stability, and split exactness - can be expressed by the property that $F$ sends a suitable class of morphisms of algebras to isomorphisms of $\mathscr{C}$.

It is known that $\operatorname{Res}_{H}^{G}\left(W_{G}\right) \subset W_{H}$ and $\operatorname{CoInd}_{H}^{G}\left(W_{H}\right) \subset W_{G}$ (see [14, Section 4.1]). Hence restriction and coinduction yield well-defined functors $\operatorname{Res}_{H}^{G}: \operatorname{KK}(G) \rightarrow \operatorname{KK}(H)$ and $\operatorname{CoInd}_{H}^{G}: \mathrm{KK}(H) \rightarrow \mathrm{KK}(G)$, which are again adjoint by the (canonical images of the) same unit and counit $\eta$ and $\epsilon$. Similarly, the projection isomorphism $\pi$ and the section $\xi$ of the counit also pass to KK. We therefore have separable monadicity $E: \operatorname{KK}(H) \stackrel{\sim}{\longrightarrow} A-\operatorname{Mod}_{\mathrm{KK}(G)}$ by Theorem 2.9 , where $A=\operatorname{CoInd}_{H}^{G}(\mathbb{C})=\mathrm{C}(G / H)$ is the $G$-algebra of continuous functions on $G / H$ equipped with its pointwise multiplication and identity. This is simply the usual underlying ring structure of $\mathrm{C}(G / H)$ as a $C^{*}$-algebra, which is isomorphic to its $\mathbb{C}$-linear dual algebra $\mathbb{C}(G / H)$ as announced in Theorem 1.2. Multiplication on $A_{H}^{G}=\mathbb{C}(G / H)$ is again given by Equation (1.4). Its separability is again guaranteed by the morphism $\sigma: A_{H}^{G} \rightarrow A_{H}^{G} \otimes A_{H}^{G}$ given by $\gamma \mapsto \gamma \otimes \gamma$.

4.5 Remark We can describe the Eilenberg-Moore equivalence more explicitly. By construction, the Eilenberg-Moore functor $E$ for the monad $\mathbb{A}=\operatorname{CoInd}_{H}^{G} \operatorname{Res}_{H}^{G}$ is given by $E(B)=\left(\operatorname{CoInd}_{H}^{G}(B), \operatorname{CoInd}_{H}^{G}\left(\epsilon_{B}\right)\right)$ for all $B \in \mathrm{KK}(H)$. Under the isomorphism of monads $\pi: A \otimes(-) \stackrel{\sim}{\longrightarrow} \mathbb{A}$, this $\mathrm{C}(G / H)$-action becomes the morphism

$$
\mathrm{C}(G / H) \otimes \operatorname{CoInd}_{H}^{G}(B) \stackrel{\pi}{\sim} \operatorname{CoInd}_{H}^{G} \operatorname{Res}_{H}^{G} \operatorname{CoInd}_{H}^{G}(B) \stackrel{\operatorname{CoInd}_{H}^{G}\left(\epsilon_{B}\right)}{\longrightarrow} \operatorname{CoInd}_{H}^{G}(B)
$$

which sends $u \otimes f$ to the function $\epsilon_{B} \circ(x \mapsto u(x) x f) \in \operatorname{CoInd}_{H}^{G}(B)$, ie, to the product $u f=(x \mapsto u(x) f(x))$. Thus the action is simply given by the pointwise multiplication of functions. The quasi-inverse $E^{-1}$ is described in Lemma 2.2. 
4.6 Remark An equivalence quite like our functor $E^{-1}$ has already been described, under the name compression, both for $G-\mathrm{C}^{*}$-algebras in [7, Lemma $\left.12.3 \mathrm{ff}\right]$ and also in the purely algebraic context of $G$-rings in [5, Section 10.3]. For $H$ a (finite) subgroup of a (countable) discrete $G$, but with $G / H$ not necessarily finite, the compression functor is defined for $G$-algebras that are "proper over $G / H$ " and yields a quasi-inverse of induction (at least at the level of algebras). Here induction refers to a certain functor from $H$-algebras to $G$-algebras which is usually not right adjoint to restriction. We suspect this induction-compression equivalence is related to our Theorem 1.2, although the details are not yet clear to us.

\section{Equivariant derived categories}

Let $G$ be a discrete group, eg a finite one, which acts on a (locally) ringed space $S=$ $\left(S, \mathcal{O}_{S}\right)$, eg a scheme. For every $g \in G$, we simply denote by $g: S \stackrel{\sim}{\longrightarrow} S$ the corresponding isomorphism of ringed spaces, which involves compatible ring isomorphisms $g^{\sharp}: \mathcal{O}_{S}(V) \stackrel{\sim}{\longrightarrow} \mathcal{O}_{S}(g V)$ for all $g \in G$ and $V \subseteq S$ open. For every sheaf $M$ on $S$, the sheaf $g^{*} M$ is given by $g^{*} M(V)=M(g V)$. For every $g_{1}, g_{2} \in G$, we have an equality $\left(g_{1} g_{2}\right)^{*}=g_{2}^{*} g_{1}^{*}$, where we could also accept an isomorphism with coherence. This equality will lighten some of the discussion below.

5.1 Definition A $G$-equivariant sheaf of $\mathcal{O}_{S}$-modules is a pair $(M, \varphi)$ where $M \in$ $\mathcal{O}_{S}-$ Mod is a sheaf of $\mathcal{O}_{S}$-modules and $\varphi=\left(\varphi_{g}\right)_{g}$ is a collection of $\mathcal{O}_{S}$-linear isomorphisms

$$
\varphi_{g}: M \stackrel{\sim}{\longrightarrow} g^{*} M
$$

indexed by $g \in G$ such that $\varphi_{g_{1} g_{2}}=g_{2}^{*}\left(\varphi_{g_{1}}\right) \circ \varphi_{g_{2}}$ for every $g_{1}, g_{2} \in G$. As usual, we often write $M$ instead of $(M, \varphi)$ and we call $\varphi$ the "action" of $G$ on $M$, keeping in mind that $G$ moves the underlying sheaf. A morphism of $G$-equivariant sheaves $f:(M, \varphi) \rightarrow\left(M^{\prime}, \varphi^{\prime}\right)$ is a morphism $f: M \rightarrow M^{\prime}$ of $\mathcal{O}_{S}$-modules such that $\varphi_{g}^{\prime} \circ f=$ $g^{*}(f) \circ \varphi_{g}$ for every $g \in G$. We denote by $\operatorname{Shv}(G ; S)$ the category of $G$-equivariant sheaves of $\mathcal{O}_{S}$-modules on $S$. It is an abelian category, with a faithful exact functor $\operatorname{Res}_{1}^{G}: \operatorname{Shv}(G ; S) \rightarrow \mathcal{O}_{S}-\operatorname{Mod}$, which forgets the $G$-equivariance.

Since $G$ is discrete, we can define the $G$-equivariant derived category of $S$ to be the derived category $\mathrm{D}(G ; S):=\mathrm{D}(\operatorname{Shv}(G ; S))$ of the above abelian category.

5.2 Remark Everywhere below, one can replace $\mathcal{O}_{S}$-modules by quasi-coherent ones (or coherent ones) if $S$ is a (noetherian) scheme. Similarly, one can put boundedness conditions on the derived categories, or conditions on the homology, etc. The 
statements remain true as long as the restriction-coinduction adjunction preserves those subcategories. Note that the ring $A_{H}^{G}$ that we are going to produce is a complex having a finite-dimensional free $\mathcal{O}_{S}$-module concentrated in degree zero; so it will usually belong to all such choices of subcategories. We leave such variations on the theme to the interested readers.

Also, the $\mathcal{O}_{S}$-module structure on the various equivariant sheaves that we will construct is not problematic, and it always comes as a second layer, once the "sheaf-part" and the " $G$-part" of the story are clear. We shall therefore emphasize the latter and leave most of the former as easy verifications.

5.3 Remark The category $\operatorname{Shv}(G ; S)$ is monoidal via the tensor product of $\mathcal{O}_{S}-$ modules and "diagonal action": modulo the identification

$$
g^{*}\left(M \otimes_{\mathcal{O}_{S}} M^{\prime}\right) \cong g^{*} M \otimes_{\mathcal{O}_{S}} g^{*} M^{\prime}
$$

one defines $\left(\varphi \otimes \varphi^{\prime}\right)_{g}$ as $\varphi_{g} \otimes \varphi_{g}^{\prime}$. This induces a left-derived tensor product on the derived category (that we do not really use in this glorious generality, since we really only need to tensor with the flat object $A_{H}^{G}$ ).

5.4 Construction Let $H \leq G$ be a subgroup. We have an obvious exact restriction functor $\operatorname{Res}_{H}^{G}: \operatorname{Shv}(G ; S) \rightarrow \operatorname{Shv}(H ; S)$ which induces the equally obvious $\operatorname{Res}_{H}^{G}: \mathrm{D}(G ; S) \rightarrow \mathrm{D}(H ; S)$. We construct the right adjoint $\operatorname{CoInd}_{H}^{G}: \operatorname{Shv}(H ; S) \rightarrow$ $\operatorname{Shv}(G ; S)$ by explicit formulas. Let $N=(N, \psi) \in \operatorname{Shv}(H ; S)$; in particular we have $\psi_{h}: N \stackrel{\sim}{\longrightarrow} h^{*} N$ for every $h \in H$. For every open $V \subseteq S$, we define the $\mathcal{O}_{S}$-module

$$
\left(\operatorname{CoInd}_{H}^{G} N\right)(V):=\left\{\left(s_{t}\right)_{t} \in \prod_{t \in G} t^{*} N(V) \mid t^{*} \psi_{h}\left(s_{t}\right)=s_{h t} \forall t \in G, h \in H\right\}
$$

whose $G$-action is given on each open by $\varphi_{g}\left(\left(s_{t}\right)_{t \in G}\right):=\left(s_{t g}\right)_{t \in G}$, observing that $s_{t g} \in(t g)^{*} N(V)=g^{*} t^{*} N(V)=t^{*} N(g V)$. It is easy to verify that CoInd ${ }_{H}^{G} N$ remains a sheaf (it is a limit), ie we do not need sheafification. The definition of CoInd $_{H}^{G}$ on morphisms is straightforward.

We define the counit $\epsilon_{N}: \operatorname{Res}_{H}^{G} \operatorname{CoInd}_{H}^{G} N \rightarrow N$ by mapping $\left(s_{t}\right)_{t \in G}$ to $s_{1} \in N(V)$ on each open $V \subseteq S$. We define the unit $\eta_{M}: M \rightarrow \operatorname{CoInd}_{H}^{G} \operatorname{Res}_{H}^{G} M$ by mapping $m \in M(V)$ to $\left(\varphi_{t}(m)\right)_{t \in G}$. These define natural transformations

$$
\eta: \operatorname{Id}_{\operatorname{Shv}(G ; S)} \longrightarrow \operatorname{CoInd}_{H}^{G} \operatorname{Res}_{H}^{G} \text { and } \quad \epsilon: \operatorname{Res}_{H}^{G} \operatorname{CoInd}_{H}^{G} \longrightarrow \operatorname{Id}_{\operatorname{Shv}(G ; S)} .
$$




\subsection{Lemma The functor}

$$
\operatorname{Res}_{H}^{G}: \operatorname{Shv}(G ; S) \rightarrow \operatorname{Shv}(H ; S)
$$

is left adjoint to

$$
\operatorname{CoInd}_{H}^{G}: \operatorname{Shv}(H ; S) \rightarrow \operatorname{Shv}(G ; S),
$$

with the above $\eta$ and $\epsilon$ as unit and counit. Moreover:

(a) The counit $\epsilon$ admits a natural section.

(b) If moreover $\mathrm{G} / \mathrm{H}$ is finite, then the adjunction satisfies the projection formula (Definition 2.7).

Proof Set $\xi: \operatorname{Id}_{\operatorname{Shv}(H ; S)} \rightarrow \operatorname{Res}_{H}^{G} \operatorname{CoInd}_{H}^{G}$ on an object $(N, \psi) \in \operatorname{Shv}(H ; S)$ and on an open $V$ to be the homomorphism $N(V) \rightarrow\left(\operatorname{CoInd}_{H}^{G} N\right)(V), n \mapsto\left(\xi(n)_{t}\right)_{t \in G}$ defined by the formula

$$
\xi(n)_{t}:=\left\{\begin{array}{cc}
\psi_{t}(n) & \text { if } t \in H \\
0 & \text { if } t \notin H .
\end{array}\right.
$$

The $H$-equivariance of this morphism is easy to check and it is clearly natural in $N$ and splits $\epsilon$, since $\xi(n)_{1}=\psi_{1}(n)=n$. This proves (a).

For the projection formula, let us assume that $G / H$ is finite. Recall that

$$
\operatorname{Res}_{1}^{G}: \operatorname{Shv}(G ; S) \rightarrow \mathcal{O}_{S}-\operatorname{Mod}
$$

detects isomorphisms. So it will suffice to prove that $\operatorname{Res}_{1}^{G}(\pi)$ is an isomorphism, where $\pi:\left(\operatorname{CoInd}_{H}^{G} N\right) \otimes M \rightarrow \operatorname{CoInd}_{H}^{G}\left(N \otimes \operatorname{Res}_{H}^{G} M\right)$ is the morphism of (2.6), and this for every $N \in \operatorname{Shv}(H ; S)$ and $M \in \operatorname{Shv}(G ; S)$. One can verify that the morphism $\pi$ is given by (the sheafification of)

$$
\begin{gathered}
\left(\operatorname{CoInd}_{H}^{G} N\right) \otimes M \\
\left(s_{t}\right)_{t \in G} \otimes m \longmapsto \operatorname{CoInd}_{H}^{G}\left(N \otimes \operatorname{Res}_{H}^{G} M\right), \\
\left(s_{t} \otimes \varphi_{t}(m)\right)_{t \in G}
\end{gathered}
$$

on simple tensors. We need to check that the above is an isomorphism of sheaves on $S$, ignoring the $G$-equivariance. Let $R \subset G$ be a full set of representatives for $G / H$. We claim that we have an isomorphism $\operatorname{proj}_{R}: \operatorname{Res}_{1}^{G} \operatorname{CoInd}_{H}^{G} \stackrel{\sim}{\longrightarrow} \prod_{r \in R} r^{*}$, which is given on every open $V \subseteq S$ by the composite of obvious inclusion and projection $\left(\operatorname{Res}_{1}^{G} \operatorname{CoInd}_{H}^{G} N\right)(V) \hookrightarrow \prod_{t \in G} t^{*} N(V) \rightarrow \prod_{r \in R} r^{*} N(V)$, ie simply by $\left(s_{t}\right)_{t \in G} \mapsto\left(s_{r}\right)_{r \in R}$ on elements. Its inverse maps $\left(s_{r}\right)_{r \in R}$ to $\left(r^{*} \psi_{h}\left(s_{r}\right)\right)_{t \in G}$ where each $t \in G$ is written in a unique way as $t=h r$ for some $h=h(t) \in H$ and some 
$r=r(t) \in R$. The defining property of the elements in $\left(\operatorname{CoInd}_{H}^{G} N\right)(V)$ shows that this is a bijection. Moreover, the following diagram of $\mathcal{O}_{S}$-modules commutes:

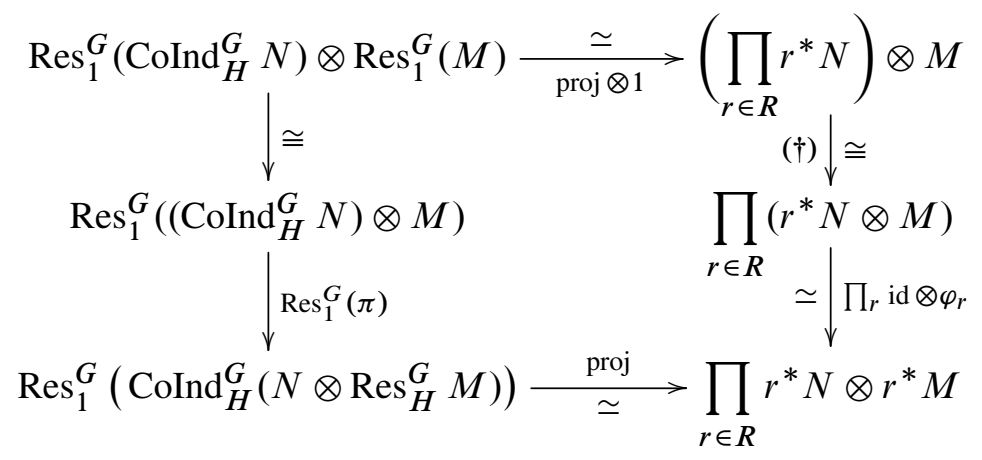

Note that the isomorphism $(\dagger)$ uses finiteness of $R \simeq G / H$ to commute product and tensor. We conclude that $\operatorname{Res}_{1}^{G}(\pi)$, hence $\pi$, is an isomorphism as wanted.

Proof of Theorem 1.3 We can use Theorem 2.9 on the sheaf level (before deriving), since its hypotheses were checked in Lemma 5.6. Let us identify the ring object $A=\operatorname{CoInd}(\mathbb{1})$, where the $\otimes$-unit $\mathbb{1}$ of $\operatorname{Shv}(H ; S)$ is $\mathcal{O}_{S}$ equipped with the trivial $H$-action: each $\psi_{h}$ is the isomorphism $h^{\sharp}: \mathcal{O}_{S} \stackrel{\sim}{\longrightarrow} h^{*} \mathcal{O}_{S}$ given with the isomorphism of ringed spaces $h: S \stackrel{\sim}{\longrightarrow} S$. Explicitly,

$$
A(V)=\left\{\left(s_{t}\right)_{t} \in \prod_{t \in G} \mathcal{O}_{S}(t V) \mid s_{h t}=h^{\sharp} s_{t} \text { for all } t \in G, h \in H\right\},
$$

where $h^{\sharp}: \mathcal{O}_{S}(t V) \stackrel{\sim}{\longrightarrow} h^{*} \mathcal{O}_{S}(t V)=\mathcal{O}_{S}(h t V)$ is as above on the open $t V$. Its multiplication (2.5) is simply mapping $\left(s_{t}\right)_{t} \otimes\left(s_{t}^{\prime}\right)_{t}$ to $\left(s_{t} s_{t}^{\prime}\right)_{t}$. To show that this ring object $A=\operatorname{CoInd}(\mathbb{1})$ is isomorphic to the announced one $A_{H}^{G}=\bigoplus_{G / H} \mathcal{O}_{S}$, we need to clarify the latter. On each open, if we name the basis $\left\{e_{\gamma}\right\}_{\gamma \in G / H}$, it is given by $A_{H}^{G}(V)=\bigoplus_{\gamma \in G / H} \mathcal{O}_{S}(V) \cdot e_{\gamma}$. The $G$-action $\varphi_{g}$ on $A_{H}^{G}$ simply maps $e_{\gamma}$ to $e_{g \gamma}$ and applies $g^{\sharp}: \mathcal{O}_{S}(V) \stackrel{\sim}{\longrightarrow} g^{*} \mathcal{O}_{S}(V)$ on the coefficients. The isomorphism $A_{H}^{G} \stackrel{\sim}{\longrightarrow} A$ can now be given on each open $V$ by mapping $e_{\gamma}$ to $\left(s_{t}\right)_{t}$, where $s_{t}=0$ if $t \notin \gamma$ and $s_{t}=1$ if $t \in \gamma$. An inverse is then given by mapping $\left(s_{t}\right)_{t} \in A(V)$ to $\sum_{\gamma \in G / H} s_{\gamma} e_{\gamma}$, where $s_{\gamma}:=\left(t^{\sharp}\right)^{-1}\left(s_{t}\right) \in \mathcal{O}_{S}(V)$ for any choice of $t \in \gamma$. The condition on $\left(s_{t}\right)_{t} \in A(V)$ shows that these coefficients $s_{\gamma}$ are well-defined. Following the above isomorphism, one sees that the multiplication on $A_{H}^{G}$ is given on the $\mathcal{O}_{S}$-basis by the now familiar formula (1.4). Separability again follows from the $A_{H}^{G}, A_{H}^{G}$-linear morphism $\sigma: A_{H}^{G} \rightarrow A_{H}^{G} \otimes A_{H}^{G}$, defined on the $\mathcal{O}_{S}$-basis by $e_{\gamma} \mapsto e_{\gamma} \otimes e_{\gamma}$.

Finally, we need to derive the adjunction. This is easy since both functors $\operatorname{Res}_{H}^{G}$ and $\operatorname{CoInd}_{H}^{G}$ are exact (the latter can be checked by postcomposing with $\operatorname{Res}_{1}^{G}$ ). Similarly, 
$A_{H}^{G} \otimes-$ is exact since $A_{H}^{G}$ is flat, the underlying $\mathcal{O}_{S}$-module being a finite sum of copies of $\mathcal{O}_{S}$. It follows that all adjunctions, units, counits, sections thereof ( $\xi$ ), etc, derive on the nose, without needing any resolution.

5.8 Remark By construction (2.1), the equivalence $E: \mathrm{D}(H ; S) \rightarrow A_{H}^{G}-\operatorname{Mod}_{\mathrm{D}(G ; S)}$ is given explicitly by $E(N)=\left(\operatorname{CoInd}_{H}^{G}(N), \operatorname{CoInd}_{H}^{G}\left(\epsilon_{N}\right)\right)$ for all $N \in \operatorname{Shv}(H ; S)$, and similarly for complexes $N \in \mathrm{D}(H ; S)$, degreewise. Under the isomorphism of monads $\pi: A_{H}^{G} \otimes(-) \stackrel{\sim}{\longrightarrow} \mathbb{A}$, this $\mathcal{O}_{S}(G / H)$-action becomes the morphism

$$
\mathcal{O}_{S}(G / H) \otimes \operatorname{CoInd}_{H}^{G}(N) \stackrel{\pi}{\longrightarrow} \operatorname{CoInd}_{H}^{G} \operatorname{Res}_{H}^{G} \operatorname{CoInd}_{H}^{G}(N) \stackrel{\operatorname{CoInd}_{H}^{G}\left(\epsilon_{N}\right)}{\longrightarrow} \operatorname{CoInd}_{H}^{G}(N) .
$$

It is given for every $\gamma \in G / H$ and every $\left(s_{t}\right)_{t} \in\left(\operatorname{CoInd}_{H}^{G} N\right)(V)$ as in (5.5) by the formula $\gamma \otimes\left(s_{t}\right)_{t} \mapsto\left(s_{t}^{\gamma}\right)_{t}$ where $s_{t}^{\gamma}=s_{t}$ when $t \in \gamma$ and zero otherwise. The quasi-inverse $E^{-1}$ can be described as in Lemma 2.2.

\section{Counterexamples}

The goal of this section is to prove Theorem 1.5. We consider a connected compact Lie group $G$ and a nontrivial finite subgroup $H \leq G$. We want to show that the restrictioncoinduction adjunction $\mathrm{SH}(G) \rightleftarrows \mathrm{SH}(H)$ is not monadic. For this, it suffices to show that the right adjoint $\mathrm{CoInd}_{H}^{G}: \mathrm{SH}(H) \rightarrow \mathrm{SH}(G)$ is not faithful. This reduces to proving that the counit $\epsilon_{D}$ : $\operatorname{Res}_{H}^{G} \operatorname{CoInd}_{H}^{G} D \rightarrow D$ is not split for some object $D \in \operatorname{SH}(H)$. This reduction is well-known: the morphism $\tau$ appearing in a distinguished triangle $\bullet \stackrel{\epsilon}{\longrightarrow} \cdot \stackrel{\tau}{\longrightarrow} \bullet \longrightarrow$ maps to zero under $\operatorname{CoInd}_{H}^{G}$ (since $\operatorname{CoInd}_{H}^{G}(\epsilon)$ is split, by the unit-counit relation) but $\tau$ is not zero (since $\epsilon$ itself is not split).

First we reduce the problem to a statement about spaces.

6.1 Lemma Let $\mathcal{C}$ and $\mathcal{D}$ be model categories in which every object is fibrant, let $d$ be a cofibrant object in $\mathcal{D}$, and let $f^{*}: \mathcal{C} \rightleftarrows \mathcal{D}: f_{*}$ be a Quillen adjunction. If the counit $\underline{\epsilon}_{d}: \mathrm{L} f^{*} \mathrm{R} f_{*} d \rightarrow d$ of the derived adjunction is split epi as a morphism in Ho $\mathcal{D}$ then the original counit $\epsilon_{d}: f^{*} f_{*} d \rightarrow d$ is split epi up to homotopy: there is a map $\xi_{d}: d \rightarrow f^{*} f_{*} d$ in $\mathcal{D}$ such that $\epsilon_{d} \circ \xi_{d}$ is homotopic to $\mathrm{id}_{d}$.

Proof If $\gamma: \Gamma c \rightarrow c$ is the cofibrant replacement then $\mathrm{L} f^{*} c=f^{*} \Gamma c$ while $\mathrm{R} f_{*} d=$ $f_{*} d$ since every object is fibrant. Recall (eg from the proof of [8, Theorem 8.5.18]) 
that the derived adjunction $\mathrm{L} f^{*}: \operatorname{Ho} \mathcal{C} \rightleftarrows \operatorname{Ho} \mathcal{D}: \mathrm{R} f_{*}$ is given as

$$
\begin{aligned}
\operatorname{Ho} \mathcal{D}\left(\mathrm{L} f^{*} c, d\right) & =\operatorname{Ho} \mathcal{D}\left(f^{*} \Gamma c, d\right)=\pi\left(\Gamma f^{*} \Gamma c, \Gamma d\right) \\
& \cong \pi\left(\Gamma f^{*} \Gamma c, d\right) \cong \pi\left(f^{*} \Gamma c, d\right) \\
& \cong \pi\left(\Gamma c, f_{*} d\right) \cong \pi\left(\Gamma c, \Gamma f_{*} d\right) \\
& =\operatorname{Ho} \mathcal{C}\left(c, f_{*} d\right)=\operatorname{Ho} \mathcal{C}\left(c, \mathrm{R} f_{*} d\right)
\end{aligned}
$$

Taking $c=\mathrm{R} f_{*} d$ and chasing the identity we find that the counit $\mathrm{L} f^{*} \mathrm{R} f_{*} d \rightarrow d$ is the homotopy class of a map $\underline{\epsilon}_{d}: \Gamma f^{*} \Gamma f_{*} d \rightarrow \Gamma d$ with the property that

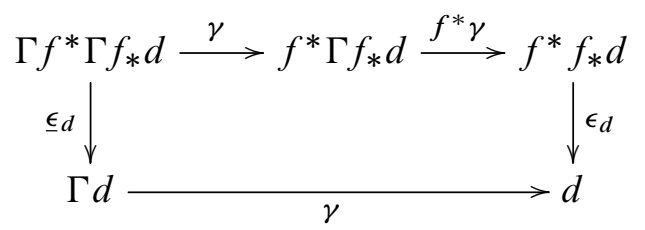

commutes up to homotopy. A section $d \rightarrow \mathrm{L} f^{*} \mathrm{R} f_{*} d$ of $\epsilon_{d}$ is the homotopy class of a map $\underline{\xi}_{d}: \Gamma d \rightarrow \Gamma f^{*} \Gamma f_{*} d$ such that $\underline{\epsilon}_{d} \circ \underline{\xi}_{d} \sim \operatorname{id} \Gamma_{d}$. If $d$ is cofibrant then the cofibrant replacement $\gamma: \Gamma d \rightarrow d$ is a homotopy equivalence. For any quasi-inverse $\delta: d \rightarrow \Gamma d$ of $\gamma$, the composite

$$
d \stackrel{\delta}{\longrightarrow} \Gamma d \stackrel{\xi d}{\longrightarrow} \Gamma f^{*} \Gamma f_{*} d \stackrel{\gamma}{\longrightarrow} f^{*} \Gamma f_{*} d \stackrel{f^{*} \gamma}{\longrightarrow} f^{*} f_{*} d
$$

defines a map $\xi_{d}: d \rightarrow f^{*} f_{*} d$ such that $\epsilon_{d} \circ \xi_{d} \sim \operatorname{id}_{d}$.

6.2 Lemma Let $H$ be a closed subgroup of a compact Lie group $G$. If $D$ is an $H-C W$ spectrum such that the counit $\epsilon_{D}$ of the restriction-coinduction adjunction $\mathrm{SH}(G) \rightleftarrows \mathrm{SH}(H)$ is a split epi as a morphism in $\mathrm{SH}(H)$, then the space-level counit $\epsilon_{\Omega^{\infty}(D)}$ is a split epi up to homotopy in the category of based $H$-spaces.

Proof Let $G \mathcal{S}$ denote the category of $G$-spectra (in the sense of [10]) so that $\mathrm{SH}(G)=$ Ho $G \mathcal{S}$ and let $G \mathcal{T}$ denote the category of based $G$-spaces. For any closed subgroup $H \leq G$, we have restriction-coinduction adjunctions at the level of spaces and at the level of spectra. In both cases, we denote coinduction by $F_{H}\left(G_{+},-\right)$. One checks immediately from the definition of $F_{H}\left(G_{+},-\right): H \mathcal{S} \rightarrow G \mathcal{S}$ [10, Section II.4, page 77] that $\Omega^{\infty} F_{H}\left(G_{+}, D\right)=F_{H}\left(G_{+}, \Omega^{\infty} D\right)$ for any $H$-spectrum $D$ and that $\Omega^{\infty}\left(\epsilon_{D}\right)=\epsilon_{\Omega^{\infty} D}$ where $\epsilon$ denotes the counit of the restriction-coinduction adjunction. If $D$ is an $H$-CW spectrum such that the derived counit $\underline{\epsilon}_{D}$ in $\operatorname{SH}(H)=\mathrm{Ho} H \mathcal{S}$ is a split epi, then Lemma 6.1 implies that the counit $\epsilon_{D}$ in $H \mathcal{S}$ is a split epi up to homotopy. The functor $\Omega^{\infty}: H \mathcal{S} \rightarrow H \mathcal{T}$ preserves homotopy and so we conclude that $\Omega^{\infty}\left(\epsilon_{D}\right)=\epsilon_{\Omega^{\infty} D}$ is split epi up to homotopy in $H \mathcal{T}$. 
6.3 Lemma Let $H$ be a closed subgroup of a connected topological group $G$, and let $X$ be a discrete based $H$-space. If there exists a continuous map $\xi: X \rightarrow$ $F_{H}\left(G_{+}, X\right)$ such that the composite

$$
X \stackrel{\xi}{\longrightarrow} F_{H}\left(G_{+}, X\right) \stackrel{\epsilon_{X}}{\longrightarrow} X
$$

is homotopic to $\mathrm{id}_{X}$ then $X$ is a trivial $H$-space.

Proof Let $x \in X$ and consider its image $\xi_{x}: G_{+} \rightarrow X$. Homotopic maps to $X$ are equal since $X$ is discrete, so $\xi_{x}(e)=\epsilon_{X}\left(\xi_{x}\right)=x$. It follows that $\xi_{x}(g)=\xi_{x}(e)=x$ for all $g \in G$ since $G$ is connected and $X$ is discrete. Hence, since $\xi_{x}$ is $H$-equivariant, $x=\xi_{x}(h)=h . \xi_{x}(e)=h . x$ for all $h \in H$.

Proof of Theorem 1.5 Armed with Lemma 6.2 and Lemma 6.3 we need only show that there exists an $H$-spectrum $D$ such that the based $H$-space $\Omega^{\infty} D$ is $H$-homotopy equivalent to a discrete based $H$-space with nontrivial $H$-action. Indeed, if $\gamma: \Gamma D \rightarrow$ $D$ denotes an $H-\mathrm{CW}$-approximation then Lemma 6.2 implies that the counit of $\Omega^{\infty} \Gamma D$ splits up to homotopy in the category of based $H$-spaces. The map $\Omega^{\infty} \gamma: \Omega^{\infty} \Gamma D \rightarrow$ $\Omega^{\infty} D$ is a weak $H$-equivalence between spaces having the $H$-homotopy type of an $H$-CW-complex and hence is an $H$-homotopy equivalence by Whitehead's theorem. Moreover, it is clear that the splitting up to homotopy of the counit for a based $\mathrm{H}$ space $X$ implies the splitting of the counit up to homotopy for any based $H$-space $H$-homotopy equivalent to $X$, so we can apply Lemma 6.3 to obtain our contradiction.

Note that we can't just take $D=\Sigma^{\infty} H_{+}$since $\Omega^{\infty} \Sigma^{\infty} H_{+}$is $Q\left(H_{+}\right)=\prod_{H} Q\left(S^{0}\right)$ rather than $H_{+}$. Nevertheless, recall that for any $H$-Mackey functor $\mathcal{M}$, there exists an equivariant Eilenberg-MacLane spectrum $\mathcal{H} \mathcal{M}$ having the property that

$$
\underline{\pi}_{q}(\mathcal{H} \mathcal{M})= \begin{cases}\mathcal{M} & \text { if } q=0 \\ 0 & \text { if } q \neq 0\end{cases}
$$

so that $\Omega^{\infty} \mathcal{H} \mathcal{M}$ is an equivariant Eilenberg-MacLane space of type $K(\mathcal{M}, 0)$. The original construction [9] is elegant but indirect (involving a Brown representability argument). A very concrete construction has been provided by $[17 ; 18]$. Applied to the "fixed point" Mackey functor $\underline{M}(H / K):=M^{K}$ associated to a $\mathbb{Z} H$-module $M$ one obtains an $H$-spectrum $\mathcal{H} \underline{M}$ whose zeroth space $\Omega^{\infty} \mathcal{H} \underline{M}$ is $H$-homotopy equivalent to $M$ regarded as a discrete based $H$-space (having 0 as the base point). Taking $M=\mathbb{Z} H$ to be the regular representation, we thus obtain a discrete nontrivial based $H$-space - provided $H$ itself is nontrivial — and this completes the proof. 


\section{References}

[1] P Balmer, Separability and triangulated categories, Adv. Math. 226 (2011) 4352-4372 MR2770453

[2] P Balmer, Separable extensions in tensor-triangular geometry and generalized Quillen stratification, preprint (2013) arXiv:1309.1808 to appear in Ann. Sci. École Norm. Sup.

[3] P Balmer, Stacks of group representations, J. Eur. Math. Soc. (JEMS) 17 (2015) 189-228 MR3312406

[4] J Bernstein, V Lunts, Equivariant sheaves and functors, Lecture Notes in Mathematics 1578, Springer, Berlin (1994) MR1299527

[5] G Cortiñas, E Ellis, Isomorphism conjectures with proper coefficients, J. Pure Appl. Algebra 218 (2014) 1224-1263 MR3168494

[6] H Fausk, P Hu, J P May, Isomorphisms between left and right adjoints, Theory Appl. Categ. 11 (2003) No. 4, 107-131 MR1988072

[7] E Guentner, $\mathbf{N}$ Higson, J Trout, Equivariant E-theory for $C^{*}$-algebras, Mem. Amer. Math. Soc. 703, Amer. Math. Soc. (2000) MR1711324

[8] PS Hirschhorn, Model categories and their localizations, Mathematical Surveys and Monographs 99, Amer. Math. Soc. (2003) MR1944041

[9] G Lewis, J P May, J McClure, Ordinary $R O(G)$-graded cohomology, Bull. Amer. Math. Soc. 4 (1981) 208-212 MR598689

[10] L G Lewis, Jr, J P May, M Steinberger, J E McClure, Equivariant stable homotopy theory, Lecture Notes in Mathematics 1213, Springer, Berlin (1986) MR866482

[11] J P May, Equivariant homotopy and cohomology theory, CBMS Regional Conference Series in Mathematics 91, Amer. Math. Soc. (1996) MR1413302

[12] J P May, The Wirthmüller isomorphism revisited, Theory Appl. Categ. 11 (2003) 132-142 MR1988073

[13] R Meyer, Equivariant Kasparov theory and generalized homomorphisms, K-Theory 21 (2000) 201-228 MR1803228

[14] R Meyer, Categorical aspects of bivariant $K$-theory, from: “ $K$-theory and noncommutative geometry”, (G Cortiñas, J Cuntz, M Karoubi, R Nest, C A Weibel, editors), Eur. Math. Soc., Zürich (2008) 1-39 MR2513331

[15] R Meyer, Universal coefficient theorems and assembly maps in KK-theory, from: "Topics in algebraic and topological $K$-theory", Lecture Notes in Math. 2008, Springer, Berlin (2011) 45-102 MR2762554

[16] R Meyer, R Nest, The Baum-Connes conjecture via localisation of categories, Topology 45 (2006) 209-259 MR2193334 
[17] PF dos Santos, A note on the equivariant Dold-Thom theorem, J. Pure Appl. Algebra 183 (2003) 299-312 MR1992051

[18] PF dos Santos, $\mathbf{Z}$ Nie, A model for equivariant Eilenberg-Mac Lane spectra arXiv: 0804.0264

Mathematics Department, University of California, Los Angeles

Los Angeles, CA 90095-1555, USA

Laboratoire de Mathématiques Paul Painlevé, Université de Lille 1

F-59665 Villeneuve-d'Ascq Cedex, France

Centre for Symmetry and Deformation, Institut for Matematiske Fag, Universitetsparken 5, DK-2100 Copenhagen, Denmark

balmer@math.ucla.edu, ivo.dellambrogio@math.univ-lille1.fr, sanders@math.ku.dk

http: //www . math.ucla.edu/ balmer,

http://math.univ-lille1.fr/ dellambr/, http://beren.blogs.ku.dk/

Received: 11 November 2014 Revised: 9 February 2015 
